\title{
Exploitation of a Klebsiella bacteriophage receptor-binding protein as a superior biorecognition molecule
}

\author{
Catarina L. Nogueira ${ }^{1,2}$, Diana P. Pires ${ }^{3}$, Rodrigo Monteiro ${ }^{3}$, Sílvio B. Santos ${ }^{3 *}$ \\ and Carla M. Carvalho ${ }^{1 *}$
}

\begin{abstract}
${ }^{1}$ International Iberian Nanotechnology Laboratory (INL), Av. Mestre José Veiga s/n, 4715-330, Braga, Portugal
${ }^{2}$ Instituto de Engenharia de Sistemas E Computadores - Microsistemas e Nanotecnologias (INESC MN), Rua Alves Redol, 9, 1000-029, Lisbon, Portugal

3 Centre of Biological Engineering, University of Minho, Campus Gualtar, 4710-057, Braga, Portugal

* corresponding authors: silviosantos@deb.uminho.pt (SBS); carla.carvalho@inl.int (CMC)
\end{abstract}

\section{Supporting Information}

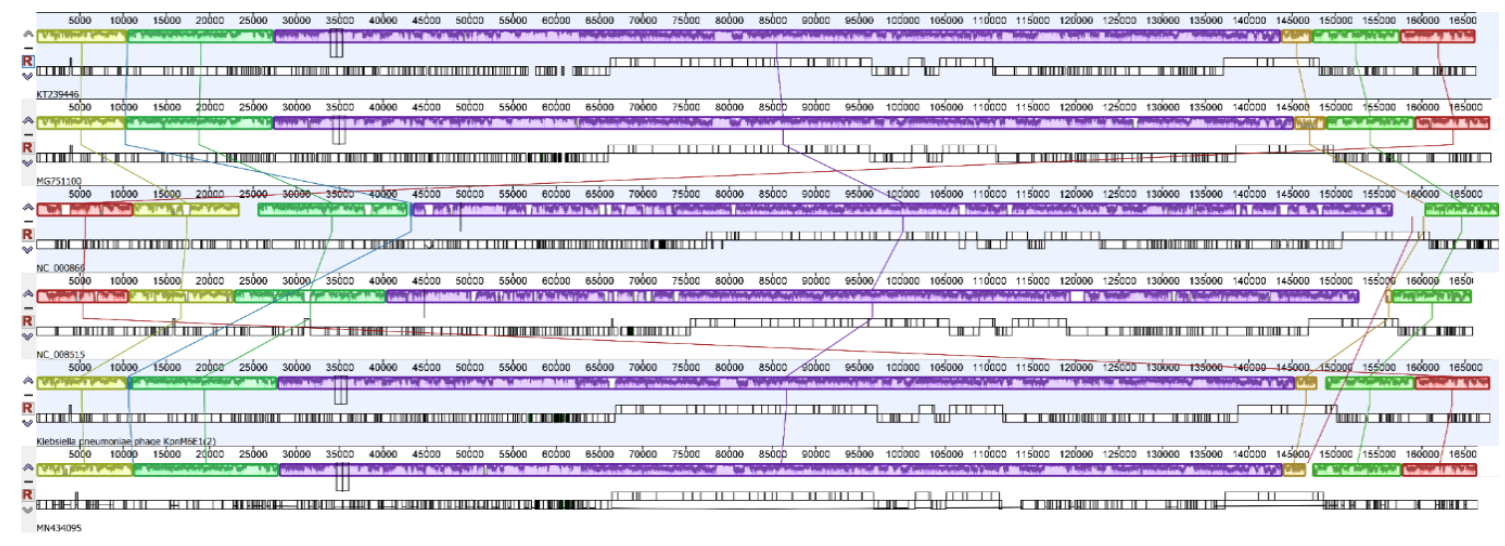

Fig. S1. Whole genome alignment of the KpnM6E1 genome and those of their closest relatives (JD18: KT239446; Kp1: MG751100; T4: NC_000866; RB32: NC_008515; Kp122: MN434095).

Table S1 - Phage KpnM6E1 infectivity assessed by the spot lysis test and binding ability of $g p 86$ analysed through fluorescence microscopy and spectrofluorometry. The fluorescence signals (a.u.) were normalized against the highest value (obtained when incubating gp86 with $\mathrm{K}$. pneumoniae strain Kp20). 


\begin{tabular}{|c|c|c|c|}
\hline Bacterial Strains & $\begin{array}{c}\text { Phage } \\
\text { Infectivity }\end{array}$ & $\begin{array}{c}g p 86 \\
\text { Fluorescence } \\
\text { Microscopy }\end{array}$ & $\begin{array}{c}g p 86 \\
\text { Spectrofluorometry }\end{array}$ \\
\hline K.pneumoniae Kp01 & - & - & 0.04 \\
\hline K.pneumoniae Kp02 & + & $+/-$ & $\mathbf{1}$ \\
\hline K.pneumoniae Kp03 & + & - & 0.04 \\
\hline K.pneumoniae Kp04 & - & + & 0.2 \\
\hline K.pneumoniae Kp05 & + & + & 0.2 \\
\hline K.pneumoniae Kp06 & + & + & 1 \\
\hline K.pneumoniae Kp07 & + & + & 0.6 \\
\hline K.pneumoniae Kp08 & + & + & 0.2 \\
\hline K.pneumoniae Kp09 & + & - & 0.03 \\
\hline K.pneumoniae Kp10 & - & + & 0.2 \\
\hline K.pneumoniae Kp11 & - & - & 0.03 \\
\hline K.pneumoniae Kp12 & - & $+/-$ & 0.2 \\
\hline K.pneumoniae Kp13 & - & + & 0.5 \\
\hline K.pneumoniae Kp14 & + & + & 0.5 \\
\hline K.pneumoniae Kp15 & + & + & 0.2 \\
\hline K.pneumoniae Kp16 & + & + & 0.3 \\
\hline K.pneumoniae Kp17 & - & $+/-$ & 0.3 \\
\hline K.pneumoniae Kp18 & - & $+/-$ & 0.4 \\
\hline K.pneumoniae Kp19 & - & $+/-$ & 0.4 \\
\hline K.pneumoniae Kp20 & - & + & 1 \\
\hline K.pneumoniae Kp21 & + & + & 1 \\
\hline K.pneumoniae Kp22 & - & - & 0 \\
\hline K.pneumoniae Kp23 & + & + & 0.4 \\
\hline K.pneumoniae Kp24 & + & + & 0.6 \\
\hline K.pneumoniae Kp25 & - & + & 0.6 \\
\hline K.pneumoniae Kp26 & + & $+/-$ & 0.5 \\
\hline K.pneumoniae Kp27 & + & $+/-$ & 0.8 \\
\hline K.pneumoniae Kp28 & + & - & 0.02 \\
\hline K.pneumoniae Kp29 & - & $+/-$ & 0.2 \\
\hline K.pneumoniae Kp30 & + & $+/-$ & 0.8 \\
\hline A.baumannii HB1 & - & - & $\mathbf{0}$ \\
\hline A.baumannii $\mathrm{HB} 2$ & - & - & $\mathbf{0}$ \\
\hline A.baumannii $\mathrm{HB} 3$ & - & - & $\mathbf{0}$ \\
\hline A.baumannii HB4 & - & - & $\mathbf{0}$ \\
\hline E.coli $\mathrm{HB} 1$ & - & - & $\mathbf{0}$ \\
\hline
\end{tabular}




\begin{tabular}{|c|c|c|c|}
\hline E.coli HB 2 & - & - & $\mathbf{0}$ \\
\hline E.coli HB 4 & - & - & $\mathbf{0}$ \\
\hline E.coli HB 5 & - & - & $\mathbf{0}$ \\
\hline E.coli HB 10 & - & - & $\mathbf{0}$ \\
\hline Enterococcus faecalis HB1 & - & - & $\mathbf{0}$ \\
\hline E. faecalis $\mathrm{HB} 2$ & - & - & $\mathbf{0}$ \\
\hline E.faecalis HB3 & - & - & $\mathbf{0}$ \\
\hline E.faecalis HB4 & - & - & $\mathbf{0}$ \\
\hline Enterococcus faecium HB5 & - & - & $\mathbf{0}$ \\
\hline E.faecium HB6 & - & - & $\mathbf{0}$ \\
\hline E.faecium HB7 & - & - & $\mathbf{0}$ \\
\hline E.faecium HB8 & - & - & $\mathbf{0}$ \\
\hline P.aeruginosa $\mathrm{HB} 1$ & - & - & $\mathbf{0}$ \\
\hline P.aeruginosa HB18 & - & - & $\mathbf{0}$ \\
\hline P.aeruginosa HB19 & - & - & $\mathbf{0}$ \\
\hline P.aeruginosa HB2 & - & - & $\mathbf{0}$ \\
\hline S.aureus HB1 & - & - & $\mathbf{0}$ \\
\hline S.aureus HB2 & - & - & $\mathbf{0}$ \\
\hline S.aureus HB3 & - & - & $\mathbf{0}$ \\
\hline S.aureus HB4 & - & - & $\mathbf{0}$ \\
\hline Staphylococcus capitis HB5 & - & - & $\mathbf{0}$ \\
\hline Staphylococcus epidermidis HB6 & - & - & $\mathbf{0}$ \\
\hline Staphylococcus equorum HB7 & - & - & $\mathbf{0}$ \\
\hline Staphylococcus haemolyticus HB8 & - & - & $\mathbf{0}$ \\
\hline Staphylococcus hominis HB9 & - & - & $\mathbf{0}$ \\
\hline Staphylococcus warneri $\mathrm{HB} 10$ & - & - & $\mathbf{0}$ \\
\hline
\end{tabular}


Table S2 - $g p 86$ amino acid sequence and primers for the amplification of $g p 86$ gene and the respective restriction enzymes.

\begin{tabular}{|c|c|c|}
\hline $\begin{array}{c}\text { Protein } \\
\text { Name }\end{array}$ & Primer Sequence $\left(5^{\prime} \rightarrow 3^{\prime}\right)^{*}$ & Amino Acid Sequence \\
\hline gp86 & $\begin{array}{c} \\
\boldsymbol{g p \mathbf { 8 6 . R v }} \\
\text { CCGCCGCTCGAGTTACGG } \\
\text { TCTATTTTACAGAATGT } \\
\text { AAGC }\end{array}$ & $\begin{array}{c}\text { MAQNNYNHYSDLAKYTIFDPTNT } \\
\text { QWPVAIKDVQSALELIGSWARTD } \\
\text { TGLPVASPTVAGVIRTATQAEVDA } \\
\text { GTIGNAAVTPATLKSTVTRPEATT } \\
\text { AVLGLTRYATNTEAAALTAGNRT } \\
\text { ITAAALGHVFKTVKAQENVDGTV } \\
\text { RLTTAAQAQAGTDETTAVTPKRV } \\
\text { VEMIGKFSVSPPSYTSATESNLGL } \\
\text { VRVATQAQVAAGAVHDGYAVTP } \\
\text { KTFMASKASDSVFGIVKFAKDSD } \\
\text { VASATSNNLAVTPKSLQALKSTK } \\
\text { DKYGLTRLSGSPTTDASLAAAAT } \\
\text { DAVFKTRRINGKTLDNDITITNNDI } \\
\text { NCYTRQESDGRYMPAGTRVGNV } \\
\text { TWVEGQSWISRGATFTCNAPWEA } \\
\text { SSRLALNVNVKFERNNDGYDNRI } \\
\text { FRFVVIVNGSQWGGELTLNIENTK } \\
\text { GGRNGHSWRFEAYASSNFFFNNIP } \\
\text { PNATVQIRPTEDSRIIFYDCMLTFC } \\
\text { TNRP }\end{array}$ \\
\hline
\end{tabular}

*Restriction endonuclease sites for SacI (forward primer) and XhoI (reverse primer) are underlined. 